\section{Symptomatische Therapie bei SJS und TEN}

Das Stevens-Johnson-Syndrom (SJS) und die toxisch epidermale Nekrolyse (TEN) sind die schwerwiegendsten Arzneimittelreaktionen, so Prof. Dr. Jean-Claude Roujeau, Paris. Die Behandlung sollte durch ein interdisziplinäres Ärzteteam erfolgen, in dem z. B. auch Augenärzte schnell Maßnahmen ergreifen können. Als Flüssigkeitssubstitution werden etwa 1,5 ml Elektrolytlösung/kg KG und 1\% betroffener Hautfläche (abgelöste oder ablösbare Haut) in 24 Stunden gegeben. Die bei SJS/TEN massiven Schmerzen sind adäquat mit Opiaten zu therapieren.

Roujeau sprach sich gegen eine rein prophylaktische Antibiose aus. Wichtig ist die Kontrolle und Therapie einer Hyperglykämie. Roujeau bevorzugt eine enterale Ernährungsbehandlung, um Stressulzera und die bakterielle Translokation aus dem Darm zu verhindern. Nach US-amerikanischen und französischen Empfehlungen sollte ein Débridement vermieden werden. Die betroffenen Hautstellen sind regelmäßig antiseptisch zu behandeln. Befallene Schleimhautareale müssen häufig (Augen bis alle zwei Stunden) befeuchtet und gepflegt werden. Wichtig sind auch Kontrolluntersuchungen vor allem der Schleimhäute etwa sechs bis acht Wo-

\section{Ende des IVIG-Mythos}

Für die Behandlung der toxischen epidermalen Nekrolyse (TEN) wurde lange die Effektivität der intravenösen Gabe von Immunglobulinen (IVIG) diskutiert und diese Therapie auch oft eingesetzt. Roujeau wies auf das "Ende des IVIG-Mythos" hin. Bereits die Arbeit von Schneck et al. im Jahr 2008 zeigte, dass im Vergleich zu einer rein symptomatischen Therapie sowohl IVIG wie auch Steroide oder die Kombination beider Therapien keinen signifikanten Vorteil aufwies [Schneck J et al. J Am Acad Dermatol 2008; 58: 33-40].

Die Anhänger der IVIG-Behandlung argumentierten, dass die Immunglobuline einen hemmenden Einfluss auf die Fas-Liganden chen nach der Entlassung, um beispielsweise Synechien rechtzeitig erkennen und behandeln zu können.

Dr. Andreas Fischer

Roujeau J.-C. Treatment recommendations. Plenary "Severe cutaneous adverse reactions"

und damit auf die für TEN charakteristische Apoptose der Keratinozyten hätten. Nach aktuelleren Daten haben aber Fas-Liganden nur eine untergeordnete Wirkung auf die Apoptose epidermaler Zellen bei TEN. In einer aktuell veröffentlichten Metaanalyse kommt eine Autorengruppe ebenfalls zu dem Schluss, dass die Datenlage "den klinischen Nutzen der IVIG nicht unterstützt ${ }^{\text {" }}$ [Huang YC et al. Br J Dermatol 2012, Mar 28, Epub ahead of print]. Roujeau empfahl, sich mehr auf das umfassende symptomatische Behandlungskonzept bei dieser Erkrankung zu konzentrieren. Dr. Andreas Fischer

Roujeau J.-C. Treatment recommendations. Plenary "Severe cutaneous adverse reactions"

\title{
Vorgehen bei Zytostatika-Hypersensitivität
}

In Hämatologie und Onkologie ist es oft problematisch, nach einer Anaphylaxie auf das auslösende Medikament generell zu verzichten. Prof. Dr. Mariana Castells von der Harvard Medical School stellte den Fall einer 18-jährigen Frau mit einem Lymphom vor, die auf die Gabe von Methotrexat wiederholt mit Bronchospastik, Flush, Blutdruckabfall sowie Absinken der Sauerstoffsättigung reagiert hatte. Nach Meinung der Hämatologen stellte diese Substanz jedoch die einzige Therapie dar, mit der eine Remission zu erzielen war. Bei der Patientin wurde ein zwölfstufiges Desensibilisierungsschema angewendet. Nur bei der letzten Applikation kam es zu einer moderaten Reaktion. Die Infusion wurde daraufhin gestoppt und nach Gabe von Steroiden, Antihistaminika und einem Leukotrieninhibitor fortgesetzt und von der Patientin toleriert. Im Anschluss erfolgte eine weitere 16-stufige Desensibilisierung. Unter einer Prämedikation (gleiche Substanzgruppen wie oben) traten diesmal keine Reaktionen mehr auf. Die Patientin unterzog sich danach einer kompletten Chemotherapie mit Remission der Erkrankung.
Desensibilisierungen können auch bei Reaktionen gegen Carboplatin oder gegen monokolonale Antikörper wie Rituximab oder Trastuzumab erfolgreich durchgeführt werden. Castells empfahl, bei der Desensibilisie- rung die vorangegangene Dosis jeweils höchstens zu verdoppeln, um eine Histaminfreisetzung zu vermeiden. Dr. Andreas Fischer

Castells M. Targeted and traditional chemotherapy. Symposium „Clinical hypersensitivity reactions to important drugs"

\section{Kutane Symptome nach Medikamentengabe}

Prof. Dr. Thomas Rustemeyer, Amsterdam, stellte Ergebnisse zur immunologischen Antwort im Rahmen verschiedener kutaner Störungen nach Medikamentengabe vor: Bei einer kontaktallergischen Reaktion auf Carbamazepin wurden periphere mononukleäre Zellen aus dem Blut isoliert. Mit diesen führte Rustemeyer Lymphozyten-Stimulationstests durch und konnten CD4-positive Zellen als reaktive T-Zellen identifizieren.

Bei Patienten mit einer toxisch epidermalen Nekrolyse (TEN) nach Einnahme von Carbamazepin dagegen zeigte die gleiche Untersuchung CD8-positive Zellen als primäre Effektor-Zellpopulation. Dieser Zelltyp konnte auch bei Diclofenac-induzierter TEN be- stimmt werden. Das nachgewiesene Interferon-gamma als stark zytotoxischer Mediator könnte die rasche Progredienz dieser Erkrankung erklären, so Rustemeyer.

In einer Untersuchung wurde der Pustelinhalt einer Patientin untersucht, die auf Minocyclin mit einer akuten generalisierten ekzematösen Pustulose (AGEP) reagiert hatte. In den neutrophilen Hautinfiltraten war Interleukin-17 nachweisbar. Möglicherweise spielen hier T-Helferzellen des Typs 17 (Th17) eine entscheidende Rolle. Dr. Andreas Fischer

Rustemeyer T. Contact allergy and drug exanthema to drugs. Symposium „Contact hypersensitivity meets drug hypersensitivity" 